\title{
BOUNDARY REGULARITY OF ADMISSIBLE OPERATORS
}

\author{
Christoph H. LAmpert
}

\begin{abstract}
In strictly pseudoconvex domains with smooth boundary, we prove a commutator relationship between admissible integral operators, as introduced by Lieb and Range, and smooth vector fields which are tangential at boundary points. This makes it possible to gain estimates for admissible operators in function spaces which involve tangential derivatives. Examples are given under with circumstances these can be transformed into genuine Sobolev- and $C^{k}$-estimates.
\end{abstract}

\section{Introduction}

In qualitative studies of the $\bar{\partial}$-equation, the $\bar{\partial}$-Neumann operator and many other topics of complex analysis explicit integral operators play an essential role. By proving uniform boundedness of their kernels in different norms, it is possible to obtain regularity results for the corresponding operators, like Hölder- or $L^{\infty}$-estimates, which usually are unavailable to the abstract $L^{2}$-theory,

While in general the analysis of integral operators involves rather delicate calculations and often the neccessity to deal with many error terms, the calculus of admissible kernels by Lieb and Range makes it possible to read off many regularity properties directly from a representation of the kernel, just by calculating its type. When studying the behaviour of admissible operators under differentiation, Lieb and Range were also able to prove Sobolev- and $C^{k}$-estimates for a subclass of kernels. Kernels which belong to this class were called admissible of commutator type, because they fulfill a commutator relationship with smooth vector fields which are tangential at boundary points. The purpose of this paper is to show that a similar commutator relationship in fact holds for all admissible kernels.

2000 Mathematics Subject Classification. 47G10, 32A25.

Key words. Integral operators, commutator relationship, $\bar{\partial}$-Neumann-problem. 
For simplicity, the calculations are only done for operators acting on functions and for domains in $\mathbb{C}^{n}$. It is however clear that the methods carry over to operators acting on forms of arbitrary degree and domains in complex manifolds. Further generalization are discussed in the last chapter.

\section{Definitions and Notation}

Let $D$ be a strictly pseudoconvex bounded domain in $\mathbb{C}^{n}$ with smooth boundary $b D$, given by a $C^{\infty}$-differentiable strictly plurisubharmonic function $\rho(\zeta)$ with $d \rho \neq 0$ on $b D$ as $D=\left\{\zeta \in \mathbb{C}^{n}: \rho(\zeta)<0\right\}$. In the product domain $D \times D$, we use coordinates $(\zeta, z)$, write $\eta^{i}:=\zeta^{i}-z^{i}$ and $\eta:=\zeta-z$ and denote derivatives of $\rho$ by indices, i. e. $\rho_{i}:=\frac{\partial}{\partial \zeta^{i}} \rho$, etc. To fix notation we will shortly repeat the definition of isotropic and admissible kernels. For an extensive introduction see [17].

Definition 1 (Isotropic kernels). Let $d s^{2}=\sum_{j, k=1}^{n} g_{j k} d \zeta^{j} d \bar{\zeta}^{k}$ be an underlying smooth hermitian metric on $\mathbb{C}^{n}$. Then by

$$
R^{2}(\zeta, z)=\sum_{j, k=1}^{n} g_{j k}\left(\zeta^{j}-z^{j}\right)\left(\bar{\zeta}^{k}-\bar{z}^{k}\right)
$$

we denote its square norm function. A smooth double differential form $\mathcal{E}(\zeta, z)$ on $\bar{D} \times \bar{D}$ is called isotropic kernel of order $m$ if there is a universal constant $M$ on $\bar{D} \times \bar{D}$ such that

$$
|\mathcal{E}(\zeta, z)| \leq M R^{m}(\zeta, z) .
$$

Although it is not strictly necessary for our results, we will assume $d s^{2}$ to be a normalized Levi-metric in order to be compatible with $[\mathbf{1 7}]$, i. e. $g_{j k}=f(\zeta) \frac{\partial^{2} r}{\partial \zeta^{j} \partial \bar{\zeta}^{k}}$ for some strictly plurisubharmonic defining function $r$ of $D$, and a smooth function $f$ chosen such that $|\partial r| \equiv 1$ on $b D$.

Definition 2 (Extended Levi polynomial). Let

$$
F(\zeta, z):=\sum_{j=1}^{n} \rho_{j}(\zeta)\left(\zeta^{j}-z^{j}\right)-\frac{1}{2} \sum_{j, k=1}^{n} \rho_{j k}(\zeta)\left(\zeta^{j}-z^{j}\right)\left(\zeta^{k}-z^{k}\right)
$$

be the Levi polynomial of $\rho$. Using a smooth patching function $\phi(\zeta, z)$ such that, for $\varepsilon>0$ sufficiently small, $\phi \equiv 1$ for $R^{2}(\zeta, z)<\frac{1}{3} \varepsilon$ and $\phi \equiv 0$ for $R^{2}(\zeta, z)>\frac{2}{3} \varepsilon$ we then obtain the extended Levi polynomial

$$
v(\zeta, z):=[-\rho(\zeta)+F(\zeta, z)] \phi(\zeta, z)+[1-\phi(\zeta, z)] R^{2}(\zeta, z) .
$$


$v$ inherits the property of being a holomorphic polynomial in $z$ of degree at most 2 in a neighbourhood of the diagonal $\Delta:=\{(z, z): z \in \bar{D}\}$, but in contrast to $F, v$ has no zeros inside of $\bar{D} \times \bar{D}$ except on the boundary diagonal $\Lambda:=\{(z, z): z \in b D\}$. In addition, from a Taylor expansion of $\rho$ it follows that $v$ is almost selfadjoint, which for us means that $v^{*}-v=\mathcal{E}_{3}$ where we write $v^{*}$ for the adjoint of $v$, i. e. $v^{*}(\zeta, z):=$ $\overline{v(z, \zeta)}$.

Definition 3 (Admissible kernels). A double differential form $\mathcal{A}(\zeta, z)$ on $\bar{D} \times \bar{D}$ is called admissible kernel, if

- $\mathcal{A}(\zeta, z)$ is smooth on $\bar{D} \times \bar{D}-\Lambda$, and

- any point in $\Lambda$ has a neighbourhood in which $\mathcal{A}$ can be given by a local representation

$$
\mathcal{A}(\zeta, z)=(-r)^{\gamma}(-\rho)^{\alpha} P^{-t_{0}} v^{t_{1}} \bar{v}^{t_{2}} v^{* t_{3}} \bar{v}^{* t_{4}} E_{m}
$$

with $r:=\rho(z)$ and $P(\zeta, z)=R^{2}(\zeta, z)+2 \rho(\zeta) r(z)$. $E_{m}$ is isotropic of order $\geq m$ for a non-negative integer $m$ and all exponents are integers, $\alpha, \gamma, t_{0}, t \geq 0$, where $t:=-\left(t_{1}+t_{2}+t_{3}+t_{4}\right)$.

A kernel is also called admissible, if it is a finite sum of kernels described above.

The admissible kernels are divided into subclasses of similar regularity by their type:

Definition 4 (Type). The type $\lambda$ of a representation as (1) is calculated as

$$
\lambda=2 n+m+\min (2, t-\alpha-\gamma)-2\left(t_{0}+t-\alpha-\gamma\right)
$$

and we call a kernel of type $\lambda$, if it has local representations of type at least $\lambda$ everywhere.

We fix the relation between integral kernels and the corresponding operators by

Definition 5 (Admissible operators). We call an operator $\boldsymbol{A}$ admissible of a certain type, if there is an admissible kernel $\mathcal{A}$ of this type such that

$$
\boldsymbol{A} f(z)=\int_{D} f(\zeta) \wedge \overline{* \mathcal{A}(\zeta, z)}
$$

where $*$ denotes the Hodge star with respect to $\zeta$ as induced by $d s^{2}$. Since the integration corresponds to the $L^{2}$-scalar product, we also write this as

$$
=(f, \mathcal{A}) .
$$


We will always use bold $\mathcal{A}$ for explicitly given admissible kernels and bold $\boldsymbol{A}$ for the corresponding operators. Lower indices indicate their types. Furthermore, we write $\mathcal{A}_{\lambda}$ (resp. $A_{\lambda}$ ) generically for a finite sum of several admissible kernels (resp. operators) of type $\lambda$, and $\varepsilon_{m}$ for general isotropic expressions of order $m$. For simplicity, we only study operators acting on functions and not on forms of arbitrary degree. General statements can then be achieved by localizing and applying the results compenentwise.

The following elementary properties of admissible operators are well known:

Lemma 1. Any admissible operator $\boldsymbol{A}_{\lambda}$ of type $\lambda \geq 1$ is a bounded linear operator

$$
\begin{aligned}
\boldsymbol{A}_{\lambda}: L^{p}(D) & \rightarrow L^{s}(D) \quad \text { for } 1 \leq p, s \leq \infty \text { and } \frac{1}{s}>\frac{1}{p}-\frac{1}{2 n+2}, \\
\boldsymbol{A}_{\lambda}: C^{0}(\bar{D}) & \rightarrow C^{0}(\bar{D}), \\
\boldsymbol{A}_{\lambda}: L^{2}(D) & \rightarrow W^{1 / 2}(D), \\
\text { where } W^{1 / 2}(D) & :=W^{\frac{1}{2}, 2}(D) \text { is the } L^{2} \text {-Sobolev space of order } \frac{1}{2} .
\end{aligned}
$$

Proof: This follows from classical non-isotropic estimates of the admissible kernels, see $[\mathbf{1 7}]$.

Admissible operators of type 0 or less are still defined -in the sense that the occuring integrals exist - but their image can contain forms of lower integrability which we will not study here.

\section{Behaviour of admissible operators under differentiation}

To control derivatives of admissible operators, estimates of the kernels themselves are not sufficient. Instead we need to study the action of vector fields on the operators' kernels. For this we introduce the following notation:

We only study vectors fields with smooth coefficients on $\bar{D}$. For any such vector field acting in the variable $z$ we write $X_{z}$. By $X_{\zeta}$ we denote the same field acting in $\zeta$. If the action is either in $z$ or $\zeta$, we use $X$ without subscript.

To express that boundary values of a vector field are contained in the (complexified real) tangent space at $b D$, we call it $T$. Since we assumed the gradient of $\rho$ not to vanish on $b D$, this implies $T_{\zeta} \rho=\mathcal{E}_{0} \rho$. For a 
vector field which at $b D$ is in the complex tangent space we write $W$, resp. $\bar{W}$ for its conjugate. Also for tangential and complex tangential vector fields we use the index convention introduced before.

Because all components of admissible kernels are smooth except at $\Lambda$, it follows that:

\section{Lemma 2.}

$$
X \mathcal{A}_{\lambda}=\mathcal{A}_{\lambda-2} \text {. }
$$

In particular, derivatives of admissible operators are again admissible operators, but of lower type. Therefore, admissible operators of high type act "smoothing" in the sense that any $\boldsymbol{A}_{\lambda}$ with $\lambda>2 k$ for $k \in \mathbb{N}$ maps $L^{p}(D) \rightarrow W^{k, p}(D)$ and $C^{0}(\bar{D}) \rightarrow C^{k}(\bar{D})$.

However, the operators most frequently occuring are only of type 1 or 2 , so in order to control higher derivatives one has to find a method to utilize the differentiabilty of the argument, e.g. by establishing a commutator relationship between the operator and the derivative acting on it. For tangential vector fields acting in the integration variable this obviously is possible:

Lemma 3. For any $\mathcal{A}_{\lambda}$ there is an $A_{\lambda}^{\prime}$ such that

$$
\left(f, T_{\zeta} \mathcal{A}_{\lambda}\right)=-\boldsymbol{A}_{\lambda} \bar{T}_{\zeta} f+A_{\lambda}^{\prime} f
$$

for all $f \in W^{1, p}(D), 1 \leq p \leq \infty$.

Proof: Because $f$ has boundary values in the Sobolev sense, we can integrate by parts and obtain

$$
\left(f, T_{\zeta} \mathcal{A}_{\lambda}\right)=-\left(\bar{T}_{\zeta} f, \mathcal{A}_{\lambda}\right)+\left(f, \mathcal{E}_{0} \mathcal{A}_{\lambda}\right) .
$$

Since $\mathcal{E}_{0} \mathcal{A}_{\lambda}$ is admissible of type $\lambda$ again, the claim follows.

However, to study regularity of $\boldsymbol{A}_{\lambda} f$, we need to apply vectorfields acting in $z$. Our main result is:

Theorem 1. Let $\boldsymbol{A}_{\lambda}$ be admissible of type $\lambda \geq 1$, let $T$ be a smooth tangential vector field. Then there is a commutator relationship

$$
T_{z} \boldsymbol{A}_{\lambda} f=\boldsymbol{A}_{\lambda} \bar{T}_{\zeta} f+A_{\lambda}^{0} f+\sum_{i=1}^{N} A_{\lambda+1}^{i} X_{\zeta}^{i} f
$$

for all $f \in W^{1, p}(D), 1 \leq p \leq \infty$, where the operators $A_{\lambda}^{0}$ and $A_{\lambda}^{i}$, $i=1, \ldots, N$ for some $N \in \mathbb{N}$ are admissible of indicated type. The vector fields $X_{\zeta}^{i}$ acting on $f$ may be non-tangential. 
The proof will be the contents of the following two sections: First we show that we only have to study so-called basic kernels, then we prove the result for them by explicit calculation.

\section{Admissible kernels with mixed singularity}

An even slightly better commutator relationship than Theorem 1 was first proved by Lieb and Range for admissible kernels of so-called commutator type, see [18]. Therefore, we do not treat those here. Instead, we first concentrate on the class of kernels for which the result is new:

Definition 6 (Kernels with mixed singularity). Let $\mathcal{A}(\zeta, z)$ be an admissible kernel given in its standard representation (1). We call $\mathcal{A}$ with mixed singularity, if $t_{1} t_{3} \geq 0, t_{2} t_{4} \geq 0$ and $\left(t_{1}+t_{3}\right)\left(t_{2}+t_{4}\right)>0$. If in addition $t_{3}=t_{4}=0$, we call $\mathcal{A}$ basic.

Being of commutator type and to have mixed singularity are mutually exclusive, because the former contains the condition $\left(t_{1}+t_{3}\right)\left(t_{2}+t_{4}\right) \leq 0$. The conditions on $t_{1} t_{3}$ and $t_{2} t_{4}$ are in fact only technical:

Lemma 4. Let $\mathcal{A}$ be admissible of type $\lambda$ and given in a local representation with $t_{1} t_{3}<0$ (resp. $\left.t_{2} t_{4}<0\right)$. Then $\mathcal{A}$ also has a representation with $t_{1} t_{3}=0\left(\right.$ resp. $\left.t_{2} t_{4}=0\right)$.

Proof: Because of $t_{1} t_{3}<0$, exactly one of the factors is positive and the other is negative. Let first be $t_{1}<0$ and $t_{3}>0$. Due to $v^{*}=v+\mathcal{E}_{3}$, any such product $v^{t_{1}} v^{* t_{3}}$ can be decomposed as

$$
v^{t_{1}} v^{* t_{3}}=\sum_{j=0}^{t_{3}} \varepsilon_{3 j} v^{t_{1}+t_{3}-j}
$$

so we obtain a representation without a power of $v^{*}$ and with leading term $v^{t_{1}+t_{3}}$, because all terms in the sum with $j \neq 0$ are even of higher type. If instead $t_{1}>0, t_{3}<0$, we use the same relation with the roles of $v$ and $v^{*}$ exchanged to obtain a representation without $v$. The case of $t_{2} t_{4}<0$ is handled analogously, using $\bar{v}^{*}=\bar{v}+\mathcal{E}_{3}$.

Thus, we only have to study representations with all $t_{i} \leq 0$, which we write more intuitively as

$$
\mathcal{A}(\zeta, z)=\frac{(-\rho)^{\alpha}(-r)^{\gamma} E_{m}}{P^{t_{0}} v^{j} \bar{v}^{k} v^{* l} \bar{v}^{* i}} .
$$


In order to obtain a commutator relationship, we now study $X_{z}+X_{\zeta}$, the combination of $X$ 's action in the $z$ variable and its action in the $\zeta$ variable. Compared to the action of $X$ alone, where we just know the elementary $X \mathcal{E}_{m}=\mathcal{E}_{m-1}$ and $X v=\mathcal{E}_{0}$, we then obtain the better

\section{Lemma 5 .}

i) $\left(X_{z}+X_{\zeta}\right) \mathcal{E}_{m}=\mathcal{E}_{m}$,

ii) $\left(X_{z}+X_{\zeta}\right) F=\mathcal{E}_{1}$,

iii) $\left(T_{z}+T_{\zeta}\right) v=\varepsilon_{1}+\varepsilon_{0} \rho$,

iv) $\left(T_{z}+T_{\zeta}\right)(v+\bar{v})=\mathcal{E}_{2}+\mathcal{E}_{0} \rho+\mathcal{E}_{0} r$,

v) $\left(X_{z}+X_{\zeta}\right) P=\varepsilon_{2}+\varepsilon_{0} \rho+\varepsilon_{0} r$.

Proof: i) follows from a Taylor expansion of the $\mathcal{E}_{m}$ term and the fact that $\left(X_{z}+X_{\zeta}\right) \eta=\mathcal{E}_{1}$, because $\frac{\partial}{\partial z_{i}} \eta=-\frac{\partial}{\partial \zeta_{i}} \eta$. ii) is a direct consequence, because $F$ is of class $\mathcal{E}_{1}$.

For iii) we first observe that due to the presence of $\varepsilon_{1}$ on the right hand side, we only have to work close to the diagonal, and since $(-\rho)$ is bounded from below by a positive constant in any relatively compact subset of $D$, even only the behaviour near the boundary diagonal matters. So, let $z_{0}$ be an arbitrary boundary point and $U=U\left(z_{0}\right)$ be a sufficiently small neighbourhood such that for $(z, \zeta) \in U \times U \cap \bar{D} \times \bar{D}$ we have $v=-\rho+F$. Then it is simple to check that

$$
\left(T_{z}+T_{\zeta}\right) v=-\left(T_{z}+T_{\zeta}\right) \rho+\left(T_{z}+T_{\zeta}\right) F=\mathcal{E}_{0} \rho+\varepsilon_{1}
$$

using the tangentiality of $T$ and part ii).

For iv) we first establish the same local situation as for equation iii). Then, from $F+\bar{F}^{*}=\sum_{j}\left(\rho_{j}-r_{j}\right) \eta^{j}+\varepsilon_{2}=\varepsilon_{2}$, we see that $v+\bar{v}^{*}=$ $\rho+r+\mathcal{E}_{2}$, and using $\bar{v}=\bar{v}^{*}+\mathcal{E}_{3}$ we obtain

$$
\begin{aligned}
\left(T_{z}+T_{\zeta}\right)(v+\bar{v}) & =\left(T_{z}+T_{\zeta}\right)\left(v+\bar{v}^{*}+\mathcal{E}_{3}\right)=\left(T_{z}+T_{\zeta}\right)\left(\rho+r+\mathcal{E}_{2}\right) \\
& =\mathcal{E}_{0} \rho+\mathcal{E}_{0} r+\mathcal{E}_{2}
\end{aligned}
$$

v) again just follows from i), since $\left(X_{z}+X_{\zeta}\right) P=\left(X_{z}+X_{\zeta}\right) \mathcal{E}_{2}+2 r X_{\zeta} \rho+$ $2 \rho X_{z} r$

From this we can derive an improved version of Lemma 2: 


\section{Lemma 6.}

$$
\left(T_{z}+T_{\zeta}\right) \mathcal{A}_{\lambda}=\mathcal{A}_{\lambda-1} .
$$

Proof: Explicit differentiation yields:

$$
\begin{aligned}
\left(T_{z}+T_{\zeta}\right) \mathcal{A}(\zeta, z)= & \left(T_{z}+T_{\zeta}\right)\left[\frac{(-\rho)^{\alpha}(-r)^{\gamma} E_{m}}{P^{t_{0}} v^{j} \bar{v}^{k} v^{* l} \bar{v}^{* m}}\right] \\
= & -\alpha T_{\zeta} \rho \frac{(-\rho)^{\alpha-1}(-r)^{\gamma} E_{m}}{P^{t_{0}} v^{j} \bar{v}^{k} v^{* l} \bar{v}^{* i}} \\
& -\gamma T_{z} r \cdot \frac{(-\rho)^{\alpha}(-r)^{\gamma-1} E_{m}}{P^{t_{0}} v^{j} \bar{v}^{k} v^{* l} \bar{v}^{* i}} \\
& +\frac{(-\rho)^{\alpha}(-r)^{\gamma}\left(T_{z}+T_{\zeta}\right) E_{m}}{P^{t_{0}} v^{j} \bar{v}^{k} v^{*} \bar{v}^{* i}} \\
& -t_{0}\left(T_{z}+T_{\zeta}\right) P \cdot \frac{(-\rho)^{\alpha}(-r)^{\gamma} E_{m}}{P^{t_{0}+1} v^{j} \bar{v}^{k} v^{* l} \bar{v}^{* i}} \\
& -j\left(T_{z}+T_{\zeta}\right) v \cdot \frac{(-\rho)^{\alpha}(-r)^{\gamma} E_{m}}{P^{t_{0}} v^{j+1} \bar{v}^{k} v^{* l} \bar{v}^{* i}} \\
& -k\left(T_{z}+T_{\zeta}\right) \bar{v} \cdot \frac{(-\rho)^{\alpha}(-r)^{\gamma} E_{m}}{P^{t_{0}} v^{j} \bar{v}^{k+1} v^{* l} \bar{v}^{* i}} \\
& -l\left(T_{z}+T_{\zeta}\right) v^{*} \cdot \frac{(-\rho)^{\alpha}(-r)^{\gamma} E_{m}}{P^{t_{0}} v^{j} \bar{v}^{k} v^{* l+1} \bar{v}^{* i}} \\
& -i\left(T_{z}+T_{\zeta}\right) \bar{v}^{*} \cdot \frac{(-\rho)^{\alpha}(-r)^{\gamma} E_{m}}{P^{t_{0}} v^{j} \bar{v}^{k} v^{* l} \bar{v}^{* i+1}} .
\end{aligned}
$$

The first four terms are in fact of type $\lambda$ because $T_{\zeta}$ and $T_{z}$ are tangential, resp. because of Lemma 5 i) and v). The other terms are all at least of type $\lambda-1$, because of Lemma 5 iii), so in total we have

$$
=\mathcal{A}_{\lambda-1} .
$$

On the level of operators this means:

Lemma 7. For any $f \in W^{1, p}(D)$ and $\lambda \geq 1$ we have

$$
T_{z} \boldsymbol{A}_{\lambda} f=\boldsymbol{A}_{\lambda} \bar{T}_{\zeta} f+A_{\lambda-1} f .
$$


Proof: Integrating Lemma 6 against $f$ we obtain

$$
\left(f,\left(T_{z}+T_{\zeta}\right) \mathcal{A}_{\lambda}\right)=A_{\lambda-1} f
$$

By applying Lemma 3 we can transfer the action of $T_{\zeta}$ onto $f$ instead of $\mathcal{A}$, and by moving $T_{z}$ out of the integral, we obtain

$$
T_{z} \boldsymbol{A}_{\lambda} f-\boldsymbol{A}_{\lambda} \bar{T}_{\zeta} f=A_{\lambda-1} f
$$

from which the claim follows.

Thus, when studying derivatives of operators $\boldsymbol{A}_{\lambda}$, all terms of type $\lambda+1$ or higher can be considered as error terms and neglected, because we can control their derivatives using Lemma 6 and the loss of 1 in type is acceptable. From the following lemma we then see that it is sufficient to study basic operators:

Lemma 8. Let $\mathcal{A}_{\lambda}$ be admissible with mixed singulariy, then there exists a basic admissible $\mathcal{A}_{\lambda}^{\prime}$ with

$$
\mathcal{A}_{\lambda}=\mathcal{A}_{\lambda}^{\prime}+\mathcal{A}_{\lambda+1}
$$

Proof: Because of $v=v^{*}+\mathcal{E}_{3}$ we have for $k \geq 1$ and any $a=a(\zeta, z)$ :

$$
\frac{a}{v^{j} v^{* l}}=\frac{v a}{v^{j+1} v^{* l}}=\frac{a}{v^{j+1} v^{* l-1}}+\frac{\mathcal{E}_{3} a}{v^{j+1} v^{* l}}
$$

and

$$
\frac{a}{\bar{v}^{k} \bar{v}^{* i}}=\frac{\bar{v} a}{\bar{v}^{k+1} \bar{v}^{* i}}=\frac{a}{\bar{v}^{k+1} \bar{v}^{* i-1}}+\frac{\mathcal{E}_{3} a}{\bar{v}^{k+1} \bar{v}^{* i}}
$$

where in both cases the last term is of type 1 higher than the others. For $\mathcal{A}_{\lambda}$ in form (3) we can apply this repeatedly in $v$ and $\bar{v}$ and obtain:

$$
\mathcal{A}_{\lambda}=\frac{(-r)^{\gamma}(-\rho)^{\alpha} E_{m}}{v^{j} \bar{v}^{k} v^{* l} \bar{v}^{* i} P^{t_{0}}}=\frac{(-r)^{\gamma}(-\rho)^{\alpha} E_{m}}{v^{j+l} \bar{v}^{k+i} P^{t_{0}}}+\mathcal{A}_{\lambda+1} .
$$

\section{Regularity of operators with mixed singularity}

For basic admissible kernels with mixed singularity we now establish the commutator relationship without a loss in type. First we prove 
Lemma 9. Let $\frac{(-r)^{\gamma}(-\rho)^{\alpha} E_{m}}{P^{t_{0}} v^{j} \bar{v}^{k}}$ be a basic kernel of type $\lambda \geq 1$ and $f \in W^{1, p}(D), 1 \leq p \leq \infty$. Then there are admissible operators $A_{\lambda+1}$ and $A_{\lambda+2}$ such that

i) $\int_{D} \frac{(-r)^{\gamma}(-\rho)^{\alpha} E_{m} f d \lambda}{P^{t_{0}} v^{j} \bar{v}^{k}}=\frac{-j}{\alpha+1} \int_{D} \frac{(-r)^{\gamma}(-\rho)^{\alpha+1} E_{m} f d \lambda}{P^{t_{0}} v^{j+1} \bar{v}^{k}}$

$$
+A_{\lambda+1} f+A_{\lambda+2} \bar{\partial} f
$$

ii) $\int_{D} \frac{(-r)^{\gamma}(-\rho)^{\alpha} E_{m} f d \lambda}{P^{t_{0}} v^{j} \bar{v}^{k}}=\frac{-k}{\alpha+1} \int_{D} \frac{(-r)^{\gamma}(-\rho)^{\alpha+1} E_{m} f d \lambda}{P^{t_{0}} v^{j} \bar{v}^{k+1}}$

$$
+A_{\lambda+1} f+A_{\lambda+2} \partial f .
$$

Since we restricted ourselves to operators acting on functions, the expressions $A_{\lambda+2} \partial f$ and $A_{\lambda+2} \bar{\partial} f$ are to be read as short-hands for a sum of operators acting on the first holomorphic resp. antiholomorphic partial derivatives of $f$.

Proof: Set $\beta:=i \partial \bar{\partial} \rho$ and define $E_{m}^{\prime}$ by the relation $E_{m}^{\prime} \partial \bar{v} \wedge \bar{\partial} v \wedge \beta^{n-1}:=$ $E_{m} d \lambda$. Then $E_{m}^{\prime}$ is indeed of class $\mathcal{E}_{m}$, because $\bar{\partial} v \wedge \partial \bar{v}$ has no zeros for $\zeta$ close to $z$ and because $\beta^{n}$ is equivalent to the Lebesgue measure $d \lambda:=* 1$. We can therefore rewrite the left hand side of i) as

$$
\int_{D} \frac{(-r)^{\gamma}(-\rho)^{\alpha} E_{m} f d \lambda}{P^{t_{0}} v^{j} \bar{v}^{k}}=\int_{D} \frac{(-r)^{\gamma}(-\rho)^{\alpha} E_{m}^{\prime} f \partial \bar{v} \wedge \bar{\partial} v \wedge \beta^{n-1}}{P^{t_{0}} v^{j} \bar{v}^{k}}
$$

and, since $\bar{\partial}(v+\rho)=\mathcal{E}_{1}$, this is

$$
=-\int_{D} \frac{(-r)^{\gamma}(-\rho)^{\alpha} E_{m}^{\prime} f \partial \bar{v} \wedge \bar{\partial} \rho \wedge \beta^{n-1}}{P^{t_{0}} v^{j} \bar{v}^{k}}+A_{\lambda+1} f .
$$


Except for $f$, the integrand is smooth with respect to $\zeta \in \bar{D}$ for any fixed $z \in D$. Since $f \in W^{1, p}$, we can integrate by parts, using a Stokeslike theorem for forms with Sobolev coefficients (see [12]) and obtain

$$
\begin{aligned}
= & \frac{-j}{\alpha+1} \int_{D} \frac{(-r)^{\gamma}(-\rho)^{\alpha+1} E_{m}^{\prime} f \partial \bar{v} \wedge \bar{\partial} v \wedge \beta^{n-1}}{P^{t_{0}} v^{j+1} \bar{v}^{k}} \\
& +\frac{1}{\alpha+1} \int_{D} \frac{(-r)^{\gamma}(-\rho)^{\alpha+1} E_{m}^{\prime} \partial \bar{v} \wedge \bar{\partial} f \wedge \beta^{n-1}}{P^{t_{0}} v^{j} \bar{v}^{k}} \\
& +\frac{1}{\alpha+1} \int_{D} \frac{(-r)^{\gamma}(-\rho)^{\alpha+1} f \partial \bar{v} \wedge \bar{\partial} E_{m}^{\prime} \wedge \beta^{n-1}}{P^{t_{0}} v^{j} \bar{v}^{k}} \\
& -\frac{k}{\alpha+1} \int_{D} \frac{(-r)^{\gamma}(-\rho)^{\alpha+1} E_{m}^{\prime} f \partial \bar{v} \wedge \bar{\partial} \bar{v} \wedge \beta^{n-1}}{P^{t_{0}} v^{j} \bar{v}^{k+1}} \\
& +\frac{1}{\alpha+1} \int_{D} \frac{(-r)^{\gamma}(-\rho)^{\alpha+1} E_{m}^{\prime} f \partial \bar{\partial} \bar{v} \wedge \beta^{n-1}}{P^{t_{0}} v^{j} \bar{v}^{k}} \\
& -\frac{t_{0}}{\alpha+1} \int_{D} \frac{(-r)^{\gamma}(-\rho)^{\alpha+1} E_{m}^{\prime} f \partial \bar{v} \wedge \bar{\partial} P \wedge \beta^{n-1}}{P^{t_{0}+1} v^{j} \bar{v}^{k}} \\
& +A_{\lambda+1} f .
\end{aligned}
$$

An additional boundary integral does not occur, because the integrand would vanish identically due to a factor $(-\rho)^{\alpha+1}$. The last four integrals are now easily identified as of type $\lambda+1$ or better (using that $\bar{\partial} \bar{v}=\mathcal{E}_{2}$ and $\left.\bar{\partial} P=\mathcal{E}_{1}+\mathcal{E}_{0} r\right)$. The term involving $\bar{\partial} f$ is always at least of type $\lambda+2$ :

$$
\begin{aligned}
= & \frac{-j}{\alpha+1} \int_{D} \frac{(-r)^{\gamma}(-\rho)^{\alpha+1} f E_{m}^{\prime} \partial \bar{v} \wedge \bar{\partial} v \wedge \beta^{n-1}}{P^{t_{0}} v^{j+1} \bar{v}^{k}} \\
& +A_{\lambda+1} f+A_{\lambda+2} \bar{\partial} f .
\end{aligned}
$$

Remembering the definition of $E_{m}^{\prime}$, part ii) of the lemma follows. Part i) is obtained from this by complex conjugation.

With Lemma 5 and 9 as tools, we are able to prove Theorem 1:

Proof of Theorem 1, for basic kernels: Let $f$ be in $W^{1, p}(D)$ and $\mathcal{A}$ basic admissible of type $\lambda \geq 1$. We only have to work in a neighbourhood of $\Lambda$ since $\mathcal{A}$ itself is smooth everywhere else. We can assume $\mathcal{A}=$ $\frac{(-r)^{\gamma}(-\rho)^{\alpha} E_{m}}{P^{t_{0}} v^{j} \bar{v}^{k}}$ in local representation and study the action of $T_{z}+T_{\zeta}$ 
on it. The corresponding calculation was already done in Lemma 6 , equation (4), so we already know that all terms where $\rho, r, E_{m}$ or $P$ are differentiated are of type $\lambda$ or higher. What remains are the terms where the differentiation falls on $v$ or $\bar{v}$ :

$$
\begin{aligned}
\left(T_{z}+T_{\zeta}\right) \mathcal{A}= & -j \frac{(-r)^{\gamma}(-\rho)^{\alpha} E_{m}\left(T_{z}+T_{\zeta}\right) v}{P^{t_{0}} v^{j+1} \bar{v}^{k}} \\
& -k \frac{(-r)^{\gamma}(-\rho)^{\alpha} E_{m}\left(T_{z}+T_{\zeta}\right) \bar{v}}{P^{t_{0}} v^{j} \bar{v}^{k+1}}+\mathcal{A}_{\lambda} .
\end{aligned}
$$

We denote the two explicit kernels on the right hand side by $\mathcal{B}^{1}(\zeta, z)$ and $\mathcal{B}^{2}(\zeta, z)$ and their corresponding operators by $\boldsymbol{B}^{1}$ and $\boldsymbol{B}^{2}$. They are both admissible of type $\lambda-1$ because of Lemma 5 iii). But in addition

$$
\begin{aligned}
\boldsymbol{B}^{1} f & =-j \int \frac{(-r)^{\gamma}(-\rho)^{\alpha} E_{m}\left(T_{z}+T_{\zeta}\right) v}{v^{j+1} \bar{v}^{k}} f d \lambda \\
& =\frac{j k}{\alpha} \int \frac{(-r)^{\gamma}(-\rho)^{\alpha+1} E_{m}\left(T_{z}+T_{\zeta}\right) v}{v^{j+1} \bar{v}^{k+1}} f d \lambda+A_{\lambda} f+A_{\lambda+1} \partial f,
\end{aligned}
$$

because of Lemma 9 ii) and similarily

$$
\begin{aligned}
\boldsymbol{B}^{2} f & =-k \int \frac{(-r)^{\gamma}(-\rho)^{\alpha} E_{m}\left(T_{z}+T_{\zeta}\right) \bar{v}}{v^{j} \bar{v}^{k+1}} f d \lambda \\
& =\frac{j k}{\alpha} \int \frac{(-r)^{\gamma}(-\rho)^{\alpha+1} E_{m}\left(T_{z}+T_{\zeta}\right) \bar{v}}{v^{j+1} \bar{v}^{k+1}} f d \lambda+A_{\lambda} f+A_{\lambda+1} \bar{\partial} f,
\end{aligned}
$$

because of Lemma 9 i). Combining these two we get

$$
\begin{aligned}
\boldsymbol{B}^{1} f+\boldsymbol{B}^{2} f= & \frac{j k}{\alpha} \int \frac{(-r)^{\gamma}(-\rho)^{\alpha+1} E_{m}\left(T_{z}+T_{\zeta}\right)(v+\bar{v})}{v^{j+1} \bar{v}^{k+1}} f d \lambda \\
& +A_{\lambda} f+A_{\lambda+1} d f
\end{aligned}
$$

where we used $d f$ for all terms in which $f$ is differentiated. Since $\left(T_{z}+\right.$ $\left.T_{\zeta}\right)(v+\bar{v})=\mathcal{E}_{2}+\mathcal{E}_{0} \rho+\mathcal{E}_{0} r$ due to Lemma $\left.5 \mathrm{iv}\right)$, the integrand is in fact admissible of type $\lambda$ as well:

$$
=A_{\lambda} f+A_{\lambda+1} d f .
$$

So we now know that

$$
\left(f,\left(T_{z}+T_{\zeta}\right) \mathcal{A}\right)=A_{\lambda} f+A_{\lambda+1} d f
$$


and in the same way as for Lemma 7 we obtain from this

$$
T_{z} \boldsymbol{A} f=\boldsymbol{A} \bar{T}_{\zeta} f+A_{\lambda} f+A_{\lambda+1} d f
$$

which in our short-hand notation is just equation (2).

As a corresponding regularity result we obtain:

Corollary 1. Let $\boldsymbol{A}$ be an admissible operator of type $\lambda \geq 1$. Let $T^{1}, \ldots, T^{k}$ be tangential vector fields. Then $T^{1} \ldots T^{k} \boldsymbol{A}$ is a bounded operator from $W^{k, p}(D)$ to $L^{p}(D)$ for $1 \leq p \leq \infty$ and from $C^{k}(\bar{D})$ to $C^{0}(\bar{D})$.

Proof: This follows by iterated use of the theorem. All $k$-th derivatives of $f$ are in $L^{p}(D)$ (resp. $C^{0}(\bar{D})$ ) and admissible operators of positive type map $L^{p}(D) \rightarrow L^{p}(D)$ and $C^{0}(\bar{D}) \rightarrow C^{0}(\bar{D})$ boundedly. Since it is well know that all admissible operators of strictly positive type act even smoothing in Sobolev and Lipschitz sense, the formulation of Corollary 1 is by far not sharp.

\section{Conclusion and further results}

We have proved that admissible operators essentially commute with tangential vectorfields in the sense that was formulated in Theorem 1. Formerly, this was only known for admissible operators of commutator type.

Our presentation was aligned with the definitions of [17]. However, the same commutator relationship holds in a wider context, including kernels with non-integer exponents and type. The condition $\lambda \geq 1$ can then be replaced by the more general $\lambda>0$. Also, the metric doesn't have to be normalized Levi. Corresponding definitions and calculations can be found in [14], where the results are used to study the Neumann problem in domains with a weighted Bergman metrics. Since all calculations are done locally, the results also easily transfer to admissible kernels in complex manifolds.

At first sight, it seems that our results cannot be applied to the more frequently used class of weighted Koppelman kernels as introducted by Berndtsson and Andersson in [4], because those are not admissible due to their singularity on the whole diagonal of $\bar{D} \times \bar{D}$ instead of just on the boundary diagonal. However, when restricting to boundary values, there is no such distinction and it is indeed often possible to treat the boundary values of Berndtsson-Andersson-kernels by the method presented here, interpreting them as boundary values of admissible kernels. Some of the following applications are also based on this. 
Further generalizations seem possible: from the proofs of the helping lemmas it becomes clear that the strict pseudoconvexity of $D$ only enters into the definition of the extended Levi polynomial $v$ which then becomes a part of the definition of admissible kernels. The actual calculation which leads to the commutator relationship would in fact be possible under much weaker assumptions, like only $v^{*}=v+\mathcal{E}_{2}$ instead of $v^{*}=v+\mathcal{E}_{3}$. It is therefore reasonable to conjecture that by a similar calculation it will be possible to prove tangential regularity for other classes of integral kernels and also in related geometrical situations where the theory of integral operators plays a role, like strictly pseudoconvex domains with only piecewise smooth boundaries $[\mathbf{2 0}],[\mathbf{2 1}]$, convex domains of finite type [7], [10], [11] or $q$-convex and $q$-concave domains $[\mathbf{1 6}],[\mathbf{1 9}]$.

\section{Applications}

To demonstrate the generality of the tangential regularity result, we present some applications from different situations, partly new, partly explaining previously known facts in a simpler manner:

- Let $\boldsymbol{A}^{b}$ be the tangential boundary values of an admissible operator $\boldsymbol{A}$ of type $\lambda \geq 1$, obtained by restricting the kernel to $b D$ (see [12]). Then $\boldsymbol{A}^{b}$ maps $C_{0 q}^{k}(\bar{D}) \rightarrow C_{0 q^{\prime}}^{k}(b D)$ for $k \in \mathbb{N} \cup\{\infty\}$ since all derivatives on $b D$ are tangential.

We can apply this for example to Cumenge's and Schuldenzucker's results about solutions to the $\bar{\partial}$-equation with boundary values in $L^{p}(b D)[\mathbf{6}],[\mathbf{2 2}]$. Since their proofs are based on admissible integral operators, it directly follows that these boundary operators preserve $C^{k}$-smoothness without the need to establish an elliptic estimate in the interior.

- Assume that the image of an admissible operator $\boldsymbol{A}$ of type $\geq 1$ is contained in $\operatorname{dom} \bar{\partial} \cap \operatorname{dom} \bar{\partial}^{*} \subset L_{0 q}^{2}(D)$, where $\bar{\partial}^{*}$ denotes the Hilbert-adjoint operator of $\bar{\partial}$. Define $\boldsymbol{A}^{b}$ as above. Then $\boldsymbol{A}^{b}$ also maps $W_{0 q}^{k}(D) \rightarrow W_{0 q^{\prime}}^{k}(b D)$.

This is due to the fact that $T^{1} \ldots T^{k} \boldsymbol{A} f$ is equal to $\boldsymbol{A} T^{1} \ldots T^{k} f$ up to error terms for any tangential vector fields $T^{1}, \ldots, T^{k}$. So, by Lemma 1 , all terms occuring are in $W_{0 q}^{1 / 2}(D)$. By the assumptions on the image of $\boldsymbol{A}$, this suffices for having an Sobolev trace in $L^{2}(b D)$, see [9] for the classical case of harmonic functions, or [8] for the general case.

- Assume $\boldsymbol{A}$ 's domain of definition to be smooth forms in the Hartogs domain $D^{\prime}:=\left\{(z, w) \in \mathbb{C}^{n} \times \mathbb{C}:|w|^{2}<r(z)\right\}$ over the base $D$ and 
define the boundary values $\boldsymbol{A}^{b}: C_{0 q}^{\infty}\left(\overline{D^{\prime}}\right) \rightarrow C_{0 q^{\prime}}^{\infty}\left(b D^{\prime}\right)$ as above. If $\boldsymbol{A}^{b}$ is invariant under rotations in $w$, then $\boldsymbol{A}^{b}$ induces an operator $\boldsymbol{A}^{\prime}: C_{0 q}^{\infty}(\bar{D}) \rightarrow C_{0 q^{\prime}}^{\infty}(\bar{D})$ using the method of going up and down in dimension. A description of this in more detail can be found in $[\mathbf{1 5}]$.

Integral kernels which derive from such a Hartogs situation were e. g. used by Andersson and Carlsson to solve the $\partial \bar{\partial}$-equation. So, in addition to the $L^{1}$-estimates which they prove in $[\mathbf{3}]$, their solution operators also preserve $C^{\infty}$-smoothness. The same method also gives rise to formulas for the canonical solution operators to $\bar{\partial}$ in weighted $L^{2}$-spaces, see [2], [1].

If additional information about derivatives are present, there are certain methods to deduce genuine $C^{k}$ - or Sobolev-estimates from the tangential ones.

- Assume that we have control over all antiholomorphic derivatives $\frac{\partial}{\partial \bar{z}_{j}}, j=1, \ldots, n$ of $\boldsymbol{A}$ by some other method. Then we have control over all $\frac{\partial}{\partial z_{j}} \boldsymbol{A}$ as well, because $\boldsymbol{A}$ essentially commutes with $Y=\sum_{j} r_{\bar{j}} \frac{\partial}{\partial z_{j}}-\sum_{j} r_{j} \frac{\partial}{\partial \bar{z}_{j}}$, see e.g. [5].

- Assume that $\boldsymbol{A}$ solves an elliptic PDE like e.g. the Neumann operator does. Then from combining the ellipticity with regularity in all tangential directions we can deduce regularity in the remaining non-tangential direction, see $[\mathbf{1 3}]$. In some cases it is possible to iterate this procedure and obtain estimates in $C^{k}(\bar{D})$-norm, see $[\mathbf{1 7}]$ for the $\bar{\partial}$-Neumann-operator itself.

\section{References}

[1] M. Andersson And J. Boo, Approximate formulas for canonical homotopy operators for the $\bar{\partial}$ complex in strictly pseudoconvex domains, Math. Scand. 87(2) (2000), 251-271.

[2] M. Andersson, J. Boo And J. Ortega-Cerdà, Canonical homotopy operators for the $\bar{\partial}$ complex in strictly pseudoconvex domains, Bull. Soc. Math. France 126(2) (1998), 245-271.

[3] M. Andersson And H. Carlsson, Formulas for approximate solutions of the $\partial \bar{\partial}$-equation in a strictly pseudoconvex domain, Rev. Mat. Iberoamericana 11(1) (1995), 67-101.

[4] B. Berndtsson And M. Andersson, Henkin-Ramirez formulas with weight factors, Ann. Inst. Fourier (Grenoble) 32(3) (1982), 91-110. 
[5] H. Boas And E. Straube, Global regularity of the $\bar{\partial}$-Neumann problem: a survey of the $L^{2}$-Sobolev theory, in: "Several complex variables" (Berkeley, CA, 1995-1996), Math. Sci. Res. Inst. Publ. 37, Cambridge Univ. Press, Cambridge, 1999, pp. 79-111.

[6] A. Cumenge, Estimations limites pour la solution canonique de l'équation $\bar{\partial} u=f$, Math. Ann. 286(4) (1990), 639-654.

[7] A. Cumenge, Sharp estimates for $\bar{\partial}$ on convex domains of finite type, Ark. Mat. 39(1) (2001), 1-25.

[8] So-Chin Chen AND MeI-Chi SHAw, "Partial differential equations in several complex variables", AMS/IP Studies in Advanced Mathematics 19, American Mathematical Society, Providence, RI; International Press, Boston, MA, 2001.

[9] B. E. J. DAhlberG, Weighted norm inequalities for the Lusin area integral and the nontangential maximal functions for functions harmonic in a Lipschitz domain, Studia Math. 67(3) (1980), 297-314.

[10] B. Fischer, $L^{p}$ estimates on convex domains of finite type, Math. Z. 236(2) (2001), 401-418.

[11] T. HeFER, Hölder and $L^{p}$ estimates for $\bar{\partial}$ on convex domains of finite type depending on Catlin's multitype, Math. Z. 242(2) (2002), $367-398$.

[12] T. Hefer, Boundary values of integral operators, Math. Nachr. $\mathbf{2 6 1 / 2 6 2}$ (2003), 85-104.

[13] J. J. Kohn And L. NirenberG, Non-coercive boundary value problems, Comm. Pure Appl. Math. 18 (1965), 443-492.

[14] C. H. LAMPERT, "Der Neumannoperator in streng pseudokonvexen Gebieten mit gewichteter Bergmanmetrik", Dissertation, Rheinische Friedrich-Wilhelms-Universität Bonn, Bonn, 2003; Bonner Mathematische Schriften 356, Universität Bonn, Mathematisches Institut, Bonn, 2003.

[15] C. H. Lampert, Going up-and-down in dimension and induced operators, Preprint, Bonn (2003).

[16] C. Laurent-ThiéBaut and J. Leiterer, Uniform estimates for the Cauchy-Riemann equation on $q$-convex wedges, Ann. Inst. Fourier (Grenoble) 43(2) (1993), 383-436.

[17] I. Lieb AND J. MiCHEL, "The Cauchy-Riemann complex. Integral formulae and Neumann problem", Aspects of Mathematics E34, Friedr. Vieweg \& Sohn, Braunschweig, 2002.

[18] I. LiEB AND R. M. RANGE, Estimates for a class of integral operators and applications to the $\bar{\partial}$-Neumann problem, Invent. Math. 85(2) (1986), 415-438. 
[19] L. Ma and S. K. Vassiliadou, $L^{p}$ estimates for the CauchyRiemann operator on $q$-convex intersections in $\mathbb{C}^{n}$, Manuscripta Math. 103(4) (2000), 413-433.

[20] J. MicheL, Randregularität des $\bar{\partial}$-Problems für stückweise streng pseudokonvexe Gebiete in $C^{n}$, Math. Ann. 280(1) (1988), 45-68.

[21] R. M. Range and Yum-Tong Siu, Uniform estimates for the $\bar{\partial}$-equation on domains with piecewise smooth strictly pseudoconvex boundaries, Math. Ann. 206 (1973), 325-354.

$[22]$ U. SCHULDENZUCKER, "Regularität der kanonischen Lösung der Cauchy-Riemannschen Differentialgleichungen in Rand- $L^{p}$ Räumen", Dissertation, Rheinische Friedrich-Wilhelms-Universität Bonn, Bonn, 1993; Bonner Mathematische Schriften 265, Universität Bonn, Mathematisches Institut, Bonn, 1994.

Mathematisches Institut

Universität Bonn

Beringstr. 6

53115 Bonn

Germany

E-mail address: chl@math.uni-bonn.de

Primera versió rebuda el 23 d'abril de 2004, darrera versió rebuda el 29 de juliol de 2004. 